\title{
THE LOCAL RANGE SET OF A MEROMORPHIC FUNCTION
}

\author{
LEON BROWN ${ }^{1}$ AND P. M. GAUTHIER ${ }^{2}$
}

\begin{abstract}
Let $f$ be a function meromorphic in a domain $G$ of the Riemann sphere. The global range set of $f$ is the set of values assumed infinitely often by $f$, and similarly the local range set of $f$ at a boundary point $p$ is the set of values assumed infinitely often in every neighborhood of $p$. Obviously any range set is a $G_{\delta}$ set. In this paper we show that every $G_{\delta}$ set is the local range set of some meromorphic function. This contrasts with the situation for the global range set. Our methods rely on prime end theory and Arakélian's approximation theorems.
\end{abstract}

Following Rudin [9], we call a set $V$ a $\Delta$-set if $V$ is a countable intersection of nested domains. In case $G$ is the unit disc, then the local and global range sets are $\Delta$-sets, and conversely it is known that every $\Delta$-set is the (local and global) range set of some function meromorphic in the unit disc (see [7], [9]).

Recently, it has been established that for an arbitrary domain, the global range set is always a $\Delta$-set [3, p. 670]. Thus if the closure of the global range set has a nonempty interior then the linear Hausdorff measure is infinite, in fact it is not even $\sigma$-finite [3, p. 670]. In this paper we study the local range set.

In $\S 1$ we extend Arakélian's approximation theorems. In $\S 2$ we examine simply connected domains and find a type of boundary point at which the local range set is always a $\Delta$-set, and a type of boundary point at which the local range set is not necessarily a $\Delta$-set. Finally we exhibit a domain having a boundary point at which any $G_{\delta}$ set can be realized as the local range set of some meromorphic function. Note that there are $G_{\delta}$ sets which are dense in the plane and of linear Hausdorff measure zero.

We shall make use of the following notations and definitions. If $G$ is a proper domain of the Riemann sphere, then $G^{*}$ denotes the onepoint compactification of $G$ and infinity will always stand for the ideal

Received by the editors January 15, 1973.

AMS (MOS) subject classifications (1970). Primary 30A72; Secondary 30A82.

Key words and phrases. Range set, cluster sets, tangential approximation.

${ }^{1}$ Supported by NSF, Grant GP 20150.

${ }^{2}$ Supported by NRC of Canada, Grant A-5597 and a grant from the Gouvernement du Québec.

(c) American Mathematical Society 1973 
point of $G^{*}$. Such topological notions as closure and complement will be with respect to the relative topology on $G$. $\partial G$ will denote the boundary of $G$ on the Riemann sphere. If $f$ is defined on $G$ and $p \in \partial G, R(f, p)$ will denote the local range set. We write $C E$ for $G \backslash E$, where $E$ will always denote a closed set.

A normal subdomain $V$ of $G$ is a domain bounded by finitely many disjoint Jordan curves and such that no component of $C V$ is precompact. A normal exhaustion $\left\{G_{n}\right\}$ of $G$ is an exhaustion such that $G_{n}$ is a normal subdomain of $G_{n+1}$ for each $n$.

If $G$ is a simply connected domain and $P$ is one of its prime ends, $I(P)$ denotes the impression of $P$. For $K$ a subset of the Riemann sphere, we write $z_{n} \rightarrow K$ if every limit point of the sequence $\left\{z_{n}\right\}$ lies in $K$. In the finite plane, $B(w, r)$ denotes the disc of center $w$ and radius $r$.

1. Approximation by holomorphic functions. In this section we recall Arakélian's fundamental theorems on approximation and extend them to suit our purpose.

Let $G$ be a proper domain of the Riemann sphere. A relatively closed set $E$ is said to be a set of uniform approximation (by functions holomorphic in $G$ ) provided that for each function $g$ holomorphic on $E^{0}$ and continuous on $E$ and each positive constant $\varepsilon$, there is a function $f$ holomorphic on all of $G$ for which

$$
|f(z)-g(z)|<\varepsilon, \quad z \in E .
$$

The following generalizes Mergelyan's celebrated theorem.

THEOREM A (ARAKéLIAN [1]). Let $G$ be a proper domain of the Riemann sphere. A (relatively) closed subset $E$ of $G$ is a set of uniform approximation by functions holomorphic in $G$, if and only if $G^{*} \backslash E$ is connected and locally connected. The reader may find expositions of Theorem $\mathrm{A}$ in [2] and [6].

We shall have need for a stronger sort of approximation than uniform approximation. A proper closed subset $E$ of $G$ is said to be a set of tangential approximation (by functions holomorphic in $G$ ) provided that for each function $g$ continuous on $E$ and holomorphic on $E^{0}$ and each positive continuous function $\varepsilon$ defined on $E$, there is a function $f$ holomorphic on all of $G$ for which

$$
|f(z)-g(z)|<\varepsilon(z), \quad z \in E .
$$

The set $E$ is said to satisfy condition A if for every neighborhood $U$ of the ideal point of $G^{*}$, there is a neighborhood $V \subset U$ of the ideal point such that no component of $E^{0}$ meets both $G^{*} \backslash U$ and $V$. 
THEOREM B (NERSESIAN [8]). Let $E$ be a (relatively) closed subset of a proper domain $G$ on the Riemann sphere. Then $E$ is a set of tangential approximation by functions holomorphic in $G$, if and only if $G^{*} \backslash E$ is connected and locally connected and $E$ satisfies condition $\mathrm{A}$.

The theory of uniform and tangential approximations has found many applications; however, in this investigation we shall need a form of approximation intermediate to these two.

An example of the type of set on which we wish to approximate is the set

$$
E=D \cap\left\{B\left(1, \frac{1}{4}\right), B\left(-1, \frac{1}{4}\right)\right\}
$$

in the unit disc $D$. This set violates condition $\mathrm{A}$ and thus tangential approximation is not possible in this case.

Let $E$ be a (relatively) closed subset of the domain $G$, and let $\varepsilon$ be a positive continuous function on $E$. The set $E$ is said to be a set of $\varepsilon$-approximation if, for each $g$ continuous on $E$ and holomorphic on $E^{0}$, there is an $f$ holomorphic on $G$ such that

$$
|f(z)-g(z)|<\varepsilon(z), \quad z \in E .
$$

Note that $E$ is a set of uniform approximation if and only if $E$ is a set of 1-approximation; $E$ is a set of tangential approximation if and only if $E$ is a set of $\varepsilon$-approximation for every positive continuous $\varepsilon$.

The following approximation theorem will serve our purpose. Moreover it is likely to have independent interest.

THEOREM 1. Let $E$ be a closed subset of a proper domain $G$ of the Riemann sphere. Suppose $G^{*} \backslash E$ is connected and locally connected. If $\varepsilon$ is a positive continuous function on $E$ such that $\varepsilon$ is constant on each component of $E^{0}$, then $E$ is a set of $\varepsilon$-approximation.

Proof. Our proof is based on Arakélian's proof as presented by W. Fuchs in [6, Theorem 3.2, p. 40]. Let $g$ be continuous on $E$ and holomorphic on $E^{0}$. In order to approximate $g$, we first observe that, by Theorem $\mathrm{A}$, there is a function $\phi$ holomorphic on $G$ such that

$$
|\log \varepsilon(z)-\phi(z)|<1, \quad z \in E .
$$

Now set $h(z)=\exp [\phi(z)-1]$. Then $h$ has no zeros and, for $z \in E$, satisfies

$$
|h(z)|=\exp [\operatorname{Re} \phi(z)-1] \leqq \exp (\log \varepsilon(z))=\varepsilon(z) .
$$

Again, by Theorem A, there is a function $f_{0}$ holomorphic on $G$ for which

$$
\left|g(z) / h(z)-f_{0}(z)\right|<1, \quad z \in E .
$$

Hence, $f=h \cdot f_{0}$ approximates $g$ within $\varepsilon$ on $E$ and the proof is complete. 
REMARK 1. The preceding proof shows that, for $G^{*} \backslash E$ connected and locally connected, $E$ is a set of $\varepsilon$-approximation if there exists a function $h$ holomorphic on $G$ and satisfying $0<|h(z)|<\varepsilon(z), z \in E$. As in the proof of Theorem 1 , we note that such an $h$ exists if there exists a function $\psi$ holomorphic on $E^{0}$ and continuous on $E$ such that $\operatorname{Re} \psi \leqq$ $\ln (\varepsilon(z)), z \in E$.

RemarK 2. Theorem B with $E^{0}=\varnothing$ which was first proved by N. V. Arakélian [1] is a consequence of Theorem 1 .

2. The local range set. The results of this section rely heavily on prime end theory. A good treatment of the theory for bounded simply connected domains is given in [4, Chapter 9]. Actually the theory holds on any proper simply connected domain of the finite plane (folklore).

THEOREM 2. Let $G$ be a proper simply connected plane domain. If a boundary point $p$ of the domain $G$ has the property

$$
p \in I(P) \Rightarrow I(P)=\{p\},
$$

for each prime end $P$, then $R(f, p)$ is a $\Delta$-set for any function $f$ meromorphic in $G$.

Proof. Let $\psi$ be a conformal map from the unit disc $D$ onto $G$, and let $K$ be the set of points of the unit circle which correspond under $\psi$ to prime ends whose impressions contain (are) $p$. Denote by $R(f \circ \psi, K)$ the set of values assumed by $f \circ \psi$ infinitely often in every neighborhood of $K$. We claim that

$$
R(f, p)=R(f \circ \psi, K) .
$$

Suppose $w$ is contained in the right member. Then there exists a sequence $\left\{z_{n}\right\}$ in $D$ such that $z_{n} \rightarrow K$ and $f \circ \psi\left(z_{n}\right)=w$. Since $K$ is compact, we may assume that $\left\{z_{n}\right\}$ converges to some point, say $e^{i \theta}$, in $K$. Now by the prime end theorem [4, Theorem 9.4, p. 173], $\left\{\psi\left(z_{n}\right)\right\}$ converges to $I\left(\psi\left(e^{i \theta}\right)\right)$ which is equal to $p$ by hypothesis. Consequently $w \in R(f, p)$. The opposite inclusion is straightforward.

To complete the proof, suppose $f$ is a function meromorphic in $G$. Let $V_{n}$ be the set of points of $D$ whose distance from $K$ is less than $1 / n$. It is not difficult to show that $V_{n}$ has only finitely many components and thus $f\left(V_{n}\right)$ is a $\Delta$-set [5] and since $R(f, K)=\bigcap_{n=1}^{\infty} f\left(V_{n}\right), R(f, K)$ is a $\Delta$-set. This follows from the deep result that the countable intersection of $\Delta$-sets is again a $\Delta$-set [3, Corollary 1]. The proof is complete.

EXAMPLE 1. We present an example to show that the condition in Theorem 2, though sufficient, is not necessary. Let $G$ be the square $0<x<3,0<y<3$ from which has been removed all segments $0<x \leqq 2$, $y=1 / n, n=1,2, \cdots$. The boundary point $p=2$ belongs to a nondegenerate 
impression, yet it is easily verified that $R(f, 2)$ is a $\Delta$-set for any $f$ meromorphic in $G$.

EXAMPLE 2. Let $G$ be the domain of Example 1. Then if $V$ is any open set, there is a function $f$, holomorphic in $G$, for which $R(f, 1)=V$.

Proof. Let $G(n)$ denote the set

$$
0<x<2, \quad 1 /(n+1)<y<1 / n, \quad n=1,2, \cdots .
$$

Let $h$ be a conformal map of the disc $(|\zeta|<1)$ onto $G$; let $H(n)$ be the domain corresponding to $G(n)$, and let $\zeta=q_{n}$ correspond to $z=1+i /(n+1)$, $n=1,2, \cdots$. Let $\varphi_{n}$ be a conformal map of $H(n)$ onto the unit disc $(|t|<1)$ such that $\zeta=q_{n}$ corresponds to $t=1$. Let $L(n)$ be the intersection of $(|t|<1)$ with a disc about $t=1$ chosen so small that

$$
\text { diameter }\left\{h\left(\varphi_{n}^{-1}(L(n))\right)\right\}<1 / n .
$$

We may write $V$ as a union of discs $V=\bigcup_{m=1}^{\infty} B\left(w_{m}, r_{m}\right)$. For each $m=1,2, \cdots$, let $\{q(m, k)\}, k=1,2, \cdots$, be a subsequence of distinct numbers of $\left\{q_{n}\right\}$ such that the set of all these subsequences constitutes a partition of $\left\{q_{n}\right\}$ into disjoint subsequences. If $q(m, k)=q_{n}$, we write $n=n(m, k)$. From this point on, when $n, m$, and $k$ appear in the same context during this proof, it is to be understood that $n=n(m, k)$.

Let $T_{n}$ be a Möbius transformation such that

$$
T_{n}(|t|<1)=B\left(w_{m}, r_{m}(1-1 /(k+1))\right),
$$

and

$$
T_{n}(L(n)) \supset \bar{B}\left(w_{m}, r_{m}(1-1 / k)\right) .
$$

Set $E=\bigcup_{n=1}^{\infty} \bar{H}(n) \cap(|\zeta|<1)$, and define $g$ on $E$ as follows: $g=T_{n} \circ \varphi_{n}$, on $\bar{H}(n) \cap(|\zeta|<1), n=1,2, \cdots$. If $\left\{\varepsilon_{n}\right\}$ is any sequence of positive numbers, then by Theorem 1 , there is a function $f_{0}$ holomorphic in $(|\zeta|<1)$ such that

$$
\left|f_{0}(\zeta)-g(\zeta)\right|<\varepsilon_{n}, \quad z \in H(n), n=1,2, \cdots .
$$

For $n=1,2, \cdots$, choose $s_{n}$ so that $\bar{B}\left(w_{m}, r_{m}(1-1 / k)\right) \subset B\left(w_{m}, s_{n}\right)$ and $\bar{B}\left(w_{m}, s_{n}\right) \subset T_{n}(L(n))$. Let $C_{n}$ be the inverse image of $\left\{\left|w_{m}-w\right|=s_{n}\right\}$ under $g$, and set $\delta_{n}=s_{n}-r_{m}(1-1 / k)$. Then $\delta_{n}>0$, and we set

$$
\varepsilon_{n}=\min \left\{\delta_{n}, r_{m}-r_{m}(1-1 /(k+1))\right\}
$$

in (4). Finally, set $f=f_{0} \circ h^{-1}$. We claim that $f$ is the desired function. First of all, from (2) and (4) it follows that the range of $f$ at $z=1$ is contained in $V$. On the other hand, it follows from (3), (4) and Rouche's 
theorem applied on $C_{n}$ that

$$
f\left(h\left(\varphi_{n}^{-1}(L(n))\right)\right) \supset B\left(w_{m}, r_{m}(1-1 / k)\right),
$$

$n=1,2, \cdots$. But (1) implies that the sets $h\left(\varphi_{n}^{-1}(L(n))\right)$ converge to $z=1$, and so $R(f, 1)$ contains each $B\left(w_{m}, r_{m}\right), m=1,2, \cdots$. That is, $R(f, 1) \supset V$ and since we have shown the opposite inclusion, the proof is complete.

The domain $G$ and the boundary point $z=1$ which we have just considered are very particular ones, however the same proof carries over to a large number of examples.

In connection with Example 2, it is of interest to present a simple example of an open set $V$ which is not a $\Delta$-set (for other examples see [5] and [9]). Let $V$ be a dense subset of the unit disc $D$ such that $V$ is a countable union of open discs whose closures are pairwise disjoint and whose diameters tend to zero. Then $V$ is not a $\Delta$-set [3, Theorem 3]. The set $\bar{D} \backslash V$ is called a Swiss cheese.

The following theorem provides a characterization of all possible range sets.

THEOREM 3. A subset $V$ of the Riemann sphere is the local range set of some meromorphic function if and only if $V$ is $a G_{\delta}$ set.

We merely sketch the proof since the essential ingredients have already been exposed in the construction of Example 2.

Proof. Obviously every range set is a $G_{\delta}$ set.

Conversely, let $V=\bigcap_{n=1}^{\infty} V(n)$, where $\{V(n)\}$ is a nested sequence of open sets. Without loss of generality, we may assume that $V$ is contained in the finite plane. Let $G$ be the square $0<x<3,0<y<3$, from which we remove the segments

$$
0<x \leqq 2, \quad y=2^{-n}+2^{-n-j}, \quad n=1,2, \cdots, j=1,2, \cdots .
$$

Set $G(n, j)=\left(0<x<2,2^{-n}+2^{-n-j-1}<y<2^{-n}+2^{n-j}\right)$. Let $h$ be a conformal map of the disc $(|\zeta|<1)$ onto $G$ and let $H(n, j)$ correspond to $G(n, j)$ under $h$. By defining an appropriate function $g$ on the union of the $G(n, j)$ 's much as in the previous example and by approximating $g$ sufficiently well on $G(n, j)$ by a function $f_{0}$ holomorphic on all of $(|\zeta|<1)$, we can obtain a function $f$ holomorphic in $G$ with the following properties:

$$
f(G(n, j)) \subset V(n), \quad j=1,2, \cdots, n=1, \cdots,
$$

and

$$
R\left(f, 1+i 2^{-n}\right)=V(n)
$$


From (5) it follows that $R(f, 1) \subset V(n), n=1,2, \cdots$, and from (6) we have that $\bigcap_{n=1}^{\infty} V(n) \subset R(f, 1)$. Thus $V=R(f, 1)$ and the proof is complete.

\section{REFERENCES}

1. N. V. Arakéljan, Uniform and tangential approximations by analytic functions, Izv. Akad. Nauk Armjan. SSR Ser. Mat. 3 (1968), no. 4-5, 273-286. (Russian) MR 43 \#530.

2. - Approximation complexe et propriétés des fonctions analytiques, Proc. Internat. Congress Math. (Nice, 1970), vol. 2, Gauthier-Villars, Paris, 1971, pp. 595600.

3. Leon Brown and P. M. Gauthier, The range set of a meromorphic function-A topological approach, J. London Math. Soc. (2) 3 (1971), 665-671. MR 44 \#5467.

4. E. F. Collingwood and A. J. Lohwater, The theory of cluster sets, Cambridge Tracts in Math. and Math. Phys., no. 56, Cambridge Univ. Press, Cambridge, 1966. MR 38 \#325.

5. H. G. Eggleston, The range set of a function meromorphic in the unit circle, Proc. London Math. Soc. (3) 5 (1955), 500-512. MR 17, 957.

6. W. H. J. Fuchs, Théorie de l'approximation des fonctions d'une variable complexe, Séminaire de Mathématiques Supérieures, no. 26 (Été, 1967), Les Presses de l'Université de Montréal, Montreal, Que., 1968. MR 41 \#5630.

7. W. Gross, Uber die Singularitäten Analytischer Funktionen, Monatsh. Math. 29 (1918), 1-47.

8. A. A. Nersesian, On the Carlesman sets, Izv. Akad. Nauk Armjan. SSR Ser. Mat. 6 (1971), 465-471. (Russian)

9. W. Rudin, On a problem of Collingwood and Cartwright, J. London Math. Soc. 30 (1955), 231-238. MR 16, 916.

Department of Mathematics, Wayne State University, Detrort, Michigan 48202

Département de Mathématique, Université de Montréal, Montréal, Québec, CANADA 